\title{
Maternal High-Fat Diet Induces Long-Lasting Defects in Bone Structure in Rat Offspring Through Enhanced Osteoclastogenesis
}

\author{
Priyanka Kushwaha ${ }^{1}$ - Seva G. Khambadkone ${ }^{2,3} \cdot$ Mengni Li ${ }^{2}$ Ethan J. Goodman ${ }^{2} \cdot$ Nandini Aravindan $^{2}$. \\ Ryan C. Riddle ${ }^{1,3,4}$ (1) $\cdot$ Kellie L. K. Tamashiro ${ }^{2,3}$
}

Received: 17 July 2020 / Accepted: 17 December 2020 / Published online: 2 January 2021

(c) The Author(s) 2021

\begin{abstract}
Maternal stressors during the prenatal and perinatal periods are associated with increased susceptibility for and severity of chronic disease phenotypes in adult offspring. In this study, we used a rat model of maternal high-fat diet (HFD) exposure during pregnancy and lactation to investigate the impact on skeletal homeostasis in offspring. In the distal femur, young male and female offspring (up to 3 weeks of age) from dams fed a HFD exhibited marked increases in trabecular bone volume relative to offspring from dams fed a chow diet, but this was followed by sustained bone loss. By 15 weeks of age, male offspring of HFD fed dams exhibited a 33\% reduction in trabecular bone volume fraction that histomorphometric analyses revealed was due to a nearly threefold increase in the abundance of bone-resorbing osteoclasts, while there were no differences between female control and HFD offspring by 15 weeks of age. The osteoblastic differentiation of male offspring-derived bone marrow stromal cells was not affected by maternal diet. However, osteoclastic precursors isolated from the male offspring of HFD fed dams exhibited enhanced differentiation in vitro, forming larger osteoclasts with higher expression of the fusion marker DC-STAMP. This effect appears to be mediated by a cell autonomous increase in the sensitivity of precursors to RANKL. Taken together, these results suggest that maternal stressors like HFD exposure have persistent consequences for the skeletal health of offspring that may ultimately lead to a predisposition for osteopenia/osteoporosis.
\end{abstract}

Keywords Maternal diet - Bone - Osteoclast - Developmental origins of health and disease

Priyanka Kushwaha and Seva G. Khambadkone have been contributed equally.

Ryan C. Riddle

rriddle1@jhmi.edu

Kellie L. K. Tamashiro

ktamashiro@jhmi.edu

1 Department of Orthopaedic Surgery, Johns Hopkins University School of Medicine, 720 Rutland Avenue, Ross 209, Baltimore, MD 21205, USA

2 Department of Psychiatry and Behavioral Sciences, Johns Hopkins University School of Medicine, 720 Rutland Avenue, Ross 618, Baltimore, MD 21205, USA

3 Cellular and Molecular Medicine Graduate Program, Johns Hopkins University School of Medicine, Baltimore, MD, USA

4 Research and Development Service, Baltimore Veterans Administration Medical Center, Baltimore, MD, USA

\section{Introduction}

Maintenance of bone mass and strength in the mammalian skeleton is orchestrated by the coordinated actions of osteoclasts responsible for the resorption of old or damaged bone and osteoblasts responsible for the deposition of new bone. Prior to the attainment of peak bone mass, osteoblastic activity matches or exceeds osteoclastic activity leading to net bone gains. With increasing age or the onset of menopause, bone resorption outpaces formation and leads to an increased risk for fragility fractures. The balances of these activities are known to be regulated by a myriad of local growth factors, hormones, and biophysical signals [1]. Likewise, genome wide association studies have identified genetic determinants for both peak bone mass and the propensity for the development of osteoporosis/osteopenia [2, $3]$.

In this study, we examined the effects of a maternal stressor on the maintenance of bone structure in offspring. The intrauterine environment is designed to support fetal development, 
but increasing epidemiologic and experimental evidence indicates that maternal stressors during this period or during early postnatal life can pattern susceptibility for or severity of disease in offspring [4, 5]. Referred to as the Developmental Origins of Health and Disease $(\mathrm{DOHaD})$ hypothesis, maternal stressors like under- or over-nutrition, trauma, or infection are associated with increased prevalence of metabolic dysfunction, cardiovascular disease, and mental illness in progeny. Though not as well studied, maternal stressors, especially those associated with nutrition, can also influence the trajectory of bone development and strength in offspring. As an example, a large cohort study ( $>50,000$ mother-child pairs) indicated that maternal consumption of a calorically dense, Western-style diet was associated with an increased risk of forearm fracture between birth and 16 years of age [6]. Conversely, consumption of a healthy diet, characterized by increased fruit and vegetable consumption and limited intake of processed food, has been associated with increased bone mineral density in offspring [7].

Studies in animal models have reported similar effects of maternal nutrition on both early skeletal development and long-term maintenance of bone structure. When skeletal homeostasis was examined in the progeny of C57BL/6 mice fed a high fat diet 4 weeks prior to conception and through weaning, a generalized decrease in mineralized tissue formation was evident at gestational day 19 [8] while femoral bone mineral density was reduced at 6 months of age [9]. Interestingly, maternal caloric restriction in this same strain also produces a reduction in bone mineral content in offspring at 6 months of age [10], suggesting that both minimal levels of nutrition and nutritional content are required for optimal skeletal health. Similar effects on early skeletal development and adult bone structure were reported by Chen and colleagues $[11,12]$ in a rat model, wherein 12 weeks of maternal high fat diet feeding also led to a down-regulation of osteogenic transcripts and increased senescence in isolated fetal calvarial osteoblasts.

Here, we challenged pregnant rats with a high-fat diet during a more limited period of fetal and postnatal development (gestation day 2 through the weaning) and examined both short and long-term effects on skeletal metabolism in offspring. Despite early increases in bone volume, the offspring of dams fed a high-fat diet exhibited significant bone loss as they approached adulthood. We demonstrate that this effect is primarily due to an increased abundance of osteoclastic cells and an increased sensitivity of osteoclast precursors to RANKL.

\section{Methods}

\section{Animals}

Pregnant female Sprague-Dawley rats were purchased from Charles River (Raleigh, NC) and received on gestation day (G) 2. Dams were randomly assigned to either a high fat diet (HFD; Research Diets D12492; 60\% kcal from fat, $20 \% \mathrm{kcal}$ from protein, $20 \% \mathrm{kcal}$ from carbohydrates; $n=13$ ) or a purified, sucrose-matched control diet (Chow; Research Diets D12450J; 10\% kcal from fat, $20 \% \mathrm{kcal}$ from protein, $70 \% \mathrm{kcal}$ from carbohydrates; $n=14)$ and individually housed in tub cages. Rats were given ad libitum access to water and maintained on a 12-h light/12-h dark cycle. Dams were maintained on their respective diets through the weaning of offspring on postnatal day $(\mathrm{P}) 21[13,14]$.

The day after birth (P1) pups were weighed and litters were culled to ten pups each (five males and five females) to standardize nutrition and maternal care across litters. To prevent litter effects from biasing our data analysis, each litter was considered $n=1$ and only one pup per sex per litter was used for each experiment. In cases where more than one pup per sex per litter was used, the data were averaged for that particular litter and counted as $n=1$. At 3 weeks of age (P21), pups were weaned, housed individually and maintained on the Chow diet for the duration of the study.

At 3 weeks of age, male and female rats were fooddeprived overnight for $16 \mathrm{~h}$ with only water available. Baseline fasted blood glucose was determined via a small tail nick by a handheld glucose meter (Freestyle; TheraSense, Alameda, CA). An oral gavage of glucose $(2.0 \mathrm{~g} /$ $\mathrm{kg}$ body wt, $20 \%$ glucose in sterile water solution) was then administered. Blood glucose was then determined using the glucometer at $15,30,60,90$, and 120 min after glucose gavage.

On P1, 3, 5 and 15 weeks of age, male and female offspring were weighed and killed by rapid decapitation. Long bone samples were collected for microCT assessment and cell culture. A separate cohort of dams $(n=6 /$ group) were euthanized at weaning (P21) after a $4 \mathrm{~h}$ fasting period during the light cycle for analysis of maternal bone architecture. Trunk blood was collected into EDTA coated tubes, centrifuged at $4{ }^{\circ} \mathrm{C}$, and plasma was collected and stored at $-80^{\circ} \mathrm{C}$ for later measurement of plasma leptin and insulin levels by ELISA (MilliporeSigma). 


\section{Skeletal Analysis}

Femur lengths were assessed at 3, 5 and 15 weeks of age with digital calipers, while P1 lengths were measured indirectly using microCT scans. Bone architectural analysis of excised, ethanol-fixed femurs was performed using a desktop microcomputed tomographic system (Sky Scan 1275 , Bruker, Belgium) in accordance with the recommendations of the American Society for Bone and Mineral Research [15]. Samples were scanned at $70 \mathrm{kV}$ and $142 \mu \mathrm{A}$ using an aluminum filter and a resolution of 9-18 $\mu \mathrm{m}$ depending on age. Scan data was reconstructed with NRecon software (Bruker, Belgium) and analyzed with CtAn Software (Bruker, Belgium). Trabecular bone architecture was assessed in a $0.5,1,1.5$, or $2.0 \mathrm{~mm}$ region of interest for P1, 3, 5 and 15 week-old offspring, respectively. Cortical parameters were examined in a $500 \mu \mathrm{m}$ region of interest centered on the femoral mid-diaphysis. Male and female offspring at each age were examined. Trabecular bone architecture in dams was assessed in a $2.0 \mathrm{~mm}$ region of interest.

Static and dynamic histomorphometric analyses were performed in 15-week-old male rats. Calcein $(5 \mathrm{mg} / \mathrm{kg})$ and Alizarin $(30 \mathrm{mg} / \mathrm{kg})$ were injected i.p. 8 and 3 days, respectively, prior to sacrifice. Excised femurs were fixed in ethanol and embedded in methyl methacrylate after passing through graded ethanol and xylene washes. Sections were cut with a Microm microtome and analyzed at standardized sites under the growth plate using a semiautomatic method (Osteoplan II; Kontron) in compliance with the guidelines of the nomenclature committee of the American Society for Bone and Mineral Research [16].

\section{Gene Expression Analyses}

Total RNA was extracted from cultured cells using Trizol (Invitrogen). One microgram of pure RNA was reverse transcribed using the iScript cDNA synthesis system (Biorad). Two microliters of cDNA was then subjected to PCR amplification using iQ SYBR Green Supermix (Bio-rad). Primer sequences were designed using Universal Probe Library ProbeFinder software (Roche Diagnostics). Reactions were normalized to endogenous $\beta$-actin transcripts. Primer sequences can be found in Table 1 .

\section{Osteoblastic Differentiation Assays}

Plastic-adherent bone marrow stromal cells were isolated from 15 -week-old male rat offspring and $10^{6}$ cells were seed to six well tissue culture plates. Osteoblastic differentiation was induced by culturing in $\alpha$ MEM supplemented with $10 \%$ FBS, $1 \%$ penicillin/streptomycin, $10^{-7} \mathrm{M}$ dexamethasone, $50 \mu \mathrm{g} / \mathrm{ml}$ ascorbic acid and $10 \mathrm{mM}$
Table 1 qPCR primer sequences

\begin{tabular}{ll}
\hline $\begin{array}{l}\text { Actb forward } \\
\text { Actb reverse }\end{array}$ & CCCGCGAGTACAACCTTCT \\
Osteoblastic genes & CGTCATCCATGGCGAACT \\
Runx2 forward & CCACAGAGCTATTAAAGTGACAGTG \\
Runx2 reverse & AACAAACTAGGTTTAGAGTCATCAAGC \\
Col1a1 forward & TCCTGGCAAGAACGGAGAT \\
Col1a1 reverse & CAGGAGGTCCACGCTCAC \\
Bglap forward & ATAGACTCCGGCGCTACCTC \\
Bglap reverse & CCAGGGGATCTGGGTAGG \\
Tnfsf11 forward & AGACACAGAAGCACTACCTGACTC \\
Tnfsf11 reverse & GGCCCCACAATGTGTTGTA \\
Tnfsf11b forward & GAGGTTTCCAGAGGACCACA \\
Tnfsf11b reverse & TGTCCATTCAATGATGTCCAA \\
Osteoclastic genes & \\
Tnfrsf11a forward & CTCGGGGTCTGGGAGTTC \\
Tnfrsf11a reverse & TGTTTCCTGTCACGTTTCCA \\
Dcstamp forward & TGTGTCCTCCCGCTGAATA \\
Dcstamp reverse & GCTTCAAAGATGGGACGATG \\
Acp5 forward & GACGGGAGAGATTGGTGATG \\
Acp5 reverse & AGTGGGAGCAGCAGGATTT \\
Ctsk forward & CGACTATCGAAAGAAAGGCTATG \\
Ctsk reverse & AAAGCCCAACAGGAACCAC \\
Oscar forward & CAGCCACTGGTCATCAGTTC \\
Oscar reverse & GAGGTTTCCCTGGGTATAGTCC \\
\hline
\end{tabular}

$\beta$-glycerophosphate. Media was replaced every $48 \mathrm{~h}$. On day 21 of in vitro differentiation, RNA samples were collected or cultures were washed with phosphate-buffered saline, fixed with ethanol, and stained with Alizarin Red-S (40 mM solution, $\mathrm{pH} 4.1$ ). After drying, Alizarin red stain was extracted in 10\% Acetic acid, neutralized with $10 \%$ Ammonium hydroxide, and optical density was measured at $405 \mathrm{~nm}$.

\section{Osteoclastic Differentiation Assays}

Bone marrow cells were isolated from the long bones of 15 -week-old male rat offspring and cultured in $\alpha$-MEM with $10 \% \mathrm{FBS}, 1 \%$ penicillin/streptomycin and $50 \mathrm{ng} /$ $\mathrm{ml}$ M-CSF for $16-18 \mathrm{~h}$ to separate adherent bone marrow stromal cells and non-adherent hematopoietic cells. The non-adherent population was collected by centrifugation and then cultured in 12 well plates in the presence of M-CSF and RANKL $(0.5-50 \mathrm{ng} / \mathrm{ml})$ for 5 days. Cultures were used for RNA isolation or fixed and stained using the TRAP-Leukocyte staining kit (Sigma). Multinucleated ( $>3$ nuclei) TRAP positive cells were counted using ImageJ software with 2-4 fields analyzed from each well. 


\section{Statistical Analyses}

Comparison between groups was performed with student $t$ test using Prism software (GraphPad). In all figures, data are expressed as mean \pm SEM. A $p$-value less than 0.05 was considered significant.

Table 2 Dams body weight, food intake and plasma hormone levels

\section{Results}

\section{HFD Feeding During Gestation and Lactation Reduces Maternal Bone Volume}

Maternal stressors like obesity or over-nutrition can produce long-lasting effects in offspring that increase susceptibility for or severity of disease phenotypes [4]. To examine long term effects of maternal diet on skeletal health in offspring, we fed pregnant dams Chow ( $10 \% \mathrm{kcal}$ from fat) or HFD (60\% kcal from fat) from day 2 of gestation (G2) through
A

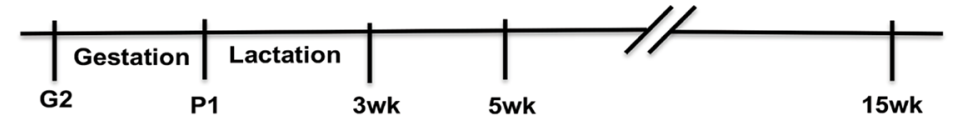

Values are shown as mean \pm SEM
$* p<0.05$

\begin{tabular}{lcc}
\hline Parameter $^{\ddagger}$ & Dam diet & \\
\cline { 2 - 3 } & Chow $(n=6)$ & High fat $(n=6)$ \\
\hline Body weight, P21 (g) & $301.3 \pm 11.5$ & $307.8 \pm 11.4$ \\
Food Intake (g), gestation + lactation & $2201.3 \pm 73.6$ & $1750.9 \pm 62.3^{*}$ \\
Caloric intake (kcal), gestation + lactation & $7374.3 \pm 246.6$ & $9174.7 \pm 326.4^{*}$ \\
Plasma leptin, P21 (ng/ml) & $3.4 \pm 0.7$ & $10.3 \pm 1.8^{*}$ \\
Plasma insulin, P21 fasted (ng/ml) & $1.9 \pm 0.3$ & $1.8 \pm 0.3$ \\
\hline
\end{tabular}

B

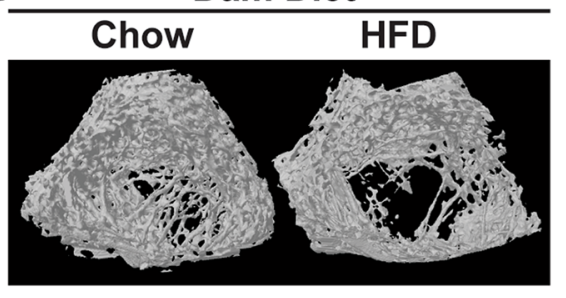

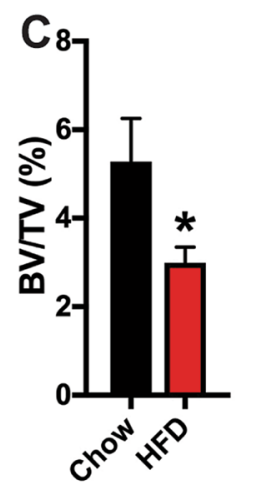
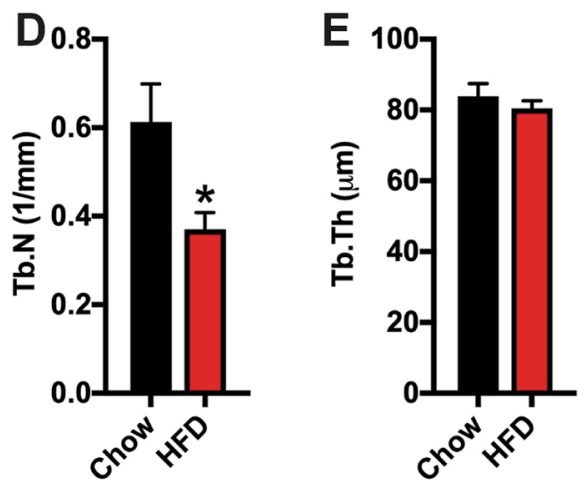

Fig. 1 High fat diet feeding during gestation and lactation exacerbates maternal bone loss. a Schematic representation of experimental strategy. Pregnant rats were fed a Chow or high fat diet (HFD) from G2 through weaning at 3 weeks of age. Offspring were weaned on to a Chow diet. b Representative 3D reconstruction of microCT exami- nation of trabecular bone structure in the distal femur of Chow and HFD dams. c-f Quantification of trabecular bone volume/tissue volume (c, BV/TV), trabecular number (d, Tb.N), trabecular thickness (e, Tb.Th) and trabecular separation (f, Tb.Sp) in the distal femur ( $n=6$ rats/group). All results are expressed as mean $\pm \mathrm{SEM}, * p<0.05$ 
weaning at 3 weeks of age (P21) [13, 14]. Dams fed the HFD exhibited reduced food intake in grams but the total caloric intake for the HFD group was significantly greater than the Chow group due to the increased caloric density of the HFD (Table 2). Fat intake and carbohydrate intake differed according to the make-up of the diet, but caloric intake from protein was well matched. Offspring were weaned and maintained on Chow diet through the conclusion of the study (Fig. 1a).

Before examining the phenotype of offspring, we examined skeletal architecture in dams at weaning. Consistent with our previous reports [17-19], there was no significant difference in body weight between dams fed a Chow or HFD on P21, but HFD dams had significantly greater plasma leptin levels with no difference in fasted insulin levels (Table 2). MicroCT analysis of the trabecular bone compartment of the distal femur revealed a more significant deterioration of trabecular structure in dams fed HFD (Fig. 1b). Relative to Chow fed dams, HFD dams exhibited a $44 \%$ reduction in trabecular bone volume with corresponding reductions in trabecular number and increases in trabecular spacing (Fig. 1c-f). Thus, HFD feeding appears to exacerbate maternal bone loss during pregnancy and lactation.

\section{Maternal HFD Feeding Alters Body Weight, Femur Length, and Glucose Homeostasis in Offspring}

As a first step in determining the effect of maternal diet on offspring, we measured body weight and femur length from birth (P1) to 15 weeks of age. At P1, body weight in the offspring of dams fed a Chow and HFD were similar (Fig. 2a, b). However, by 3 weeks of age, both male and female offspring of HFD dams exhibited a $20 \%$ increase in body weight relative to offspring of Chow dams. Body weight normalized by 5 weeks of age, but male offspring of HFD dams developed an increase in body weight again by 15 weeks of age. These data are consistent with our previous findings [13].

Femur length in offspring of HFD dams were comparable to those of Chow dams (Fig. 2c, d) during the period of most rapid longitudinal growth (0-5 weeks of age, [20]). By skeletal maturity, male offspring of HFD dams exhibited a $5 \%$ decrease in femur length relative to offspring of Chow dams (Fig. 2c). Though not directly examined here, previous studies indicate that longitudinal growth between 5 weeks of age and maturity in rats is primarily due to changes in the rate of chondrocyte proliferation and alterations in cell volume [20, 21].

Assessment of fasting blood glucose levels and the performance of oral glucose tolerance testing at 3 weeks of age revealed that maternal HFD at least temporarily impaired glucose homeostasis in offspring. Fasting blood glucose levels were significantly increased in the female offspring of HFD dams (Fig. 2g), while a strong upward trend was evident in their male littermates ( $p=0.07$, Fig. 2e). Both male and female offspring of HFD dams exhibited impairments in glucose disposal during glucose tolerance testing as blood glucose levels peaked higher than the offspring of Chow dams after oral glucose administration (Fig. 2f, h). Our previous studies indicate the impairment in glucose tolerance recovers with age due to an increase in glucose-stimulated insulin secretion [17-19]. Taken together these data indicate that maternal high fat diet feeding during gestation and the lactation periods exerts long term effects on metabolism and longitudinal growth, especially in male offspring.

\section{Maternal HFD Feeding Induces Age-Related Defects in Trabecular Bone Volume in Offspring}

We next utilized microCT analyses to examined the effects of maternal nutrition on skeletal homeostasis. In male neonates (P1), offspring from HFD dams exhibited a robust increase in femoral trabecular volume (Fig. 3a-e), secondary to increases in trabecular number and trabecular thickness, relative to offspring from Chow dams. The increase in bone volume persisted through 3 weeks of age (Fig. $3 \mathrm{f}-\mathrm{j}$ ), but was followed by a more rapid rate of bone loss than that evident in offspring of Chow dams. By 15 weeks of age, trabecular bone volume in offspring of HFD dams was significantly reduced $(\sim 33 \%)$ relative to that of offspring from Chow dams. No difference in cortical bone parameters were evident at any of these timepoints.

Female neonates (P1) from HFD dams exhibited similarly robust increases in femoral trabecular bone volume, primarily due to an increase in trabecular thickness, when compared to offspring of Chow dams (Fig. 4a-e). As with male offspring, the increase in trabecular bone volume remained evident at 3 weeks of age in female offspring of HFD dams (Fig. $4 \mathrm{f}-\mathrm{j}$ ), but at the later timepoints ( 5 and 15 weeks) trabecular bone volume was equivalent in the two groups. These data indicated that female offspring of HFD dams also exhibit a more rapid rate of bone loss, but the effect is limited to the period between 3 and 5 weeks of age. Again, no difference in cortical bone parameters were evident at any timepoint in female offspring.

To elucidate the cellular basis for the more dramatic trabecular bone loss in offspring of HFD dams, we performed static and dynamic histomorphometric analyses in 15-week-old offspring. We focused on male offspring since this sex exhibited the most severe phenotype. Parameters of bone formation in offspring of HFD dams were not significantly different from the offspring of Chow dams, though modest increases in osteoid volume per bone volume, osteoid surface per bone surface, osteoblast numbers per bone perimeter, and mineralizing surface per bone surface were evident (Table 3). Rather, offspring of 
Fig. 2 Effect of maternal diet on body weight and femur length in offspring. Body weight of male (a) and female (b) offspring were assessed on P1 (postnatal week 0) and at the indicated timepoints through 15 weeks of age $(n=8-15$ rats/ group). Femur length of male (c) and female (d) offspring were assessed at each timepoint ( $n=8-15$ rats/group). Glucose homeostasis was assessed by measuring fasting blood glucose $(\mathbf{e}, \mathbf{g})$ and performing oral glucose tolerance testing $(\mathbf{f}, \mathbf{h}$ in male (e-f) and female (g, h) offspring at 3 weeks of age ( $n=8$ rats/group). All results are expressed as mean \pm SEM, $* p<0.05$
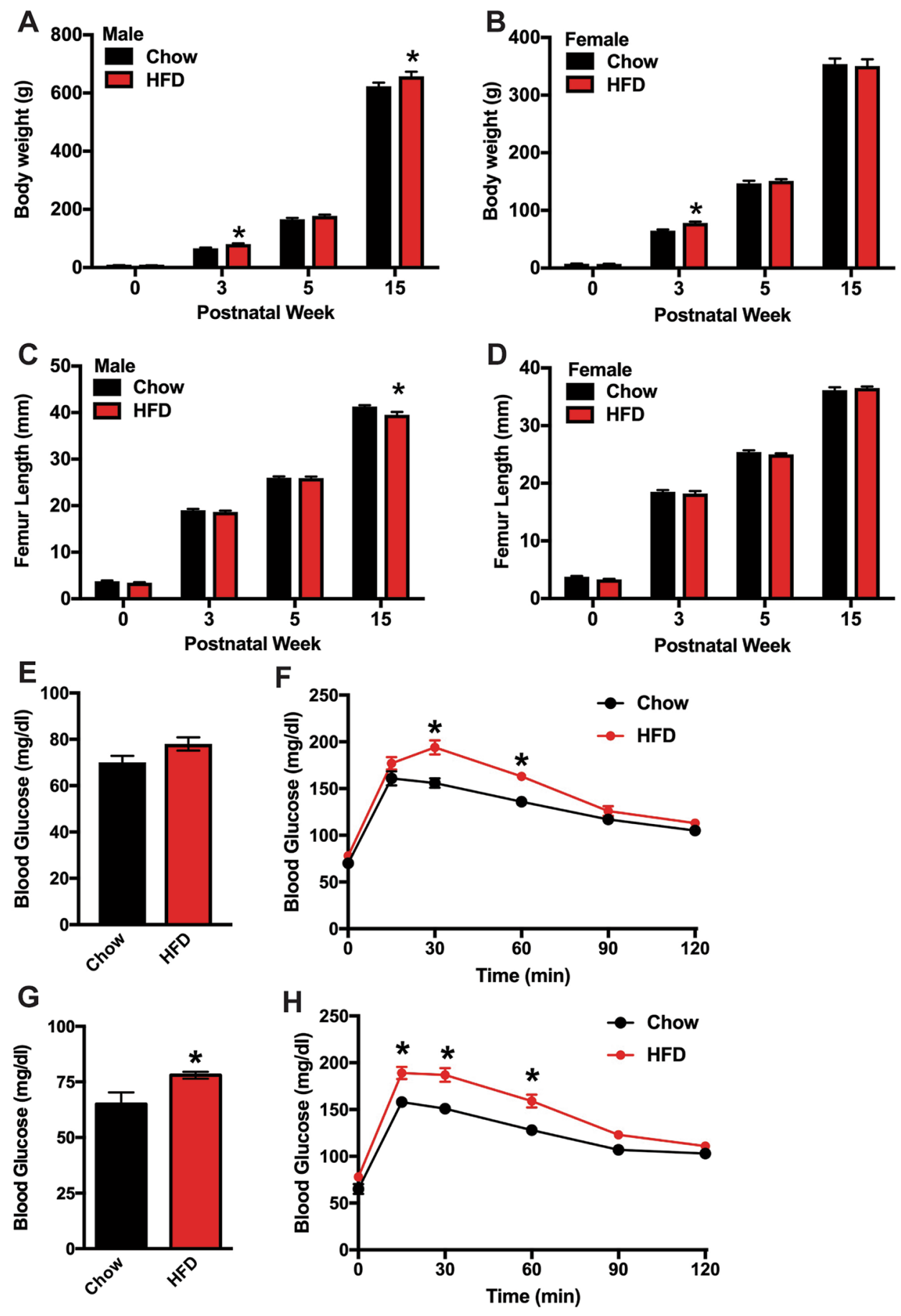

Time (min)

HFD dams exhibited marked increases in parameters of bone resorption. Most notably, osteoclast number per bone perimeter was nearly 3 times higher in offspring of HFD dams when compared to those from Chow dams. Therefore, maternal HFD feeding during gestation and lactation induces a state of heightened bone turnover weighted toward resorption that results in significant deficiencies in bone structure as progeny approach adulthood.

\section{Maternal HFD Feeding Enhances RANKL Sensitivity in Bone Marrow Macrophages of Male Offspring}

To complement the histomorphometric studies, we examined the in vitro osteoblastic and osteoclastic differentiation potential of bone marrow-derived cells. Consistent with the in vivo bone formation parameters (Table 3), bone marrow stromal cell cultures isolated from male offspring of Chow 
A

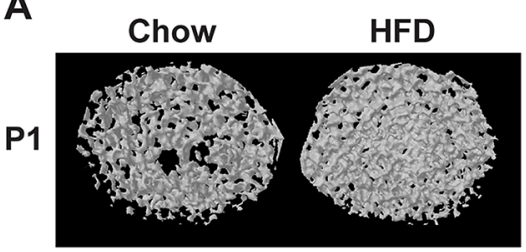

$\mathbf{F}$

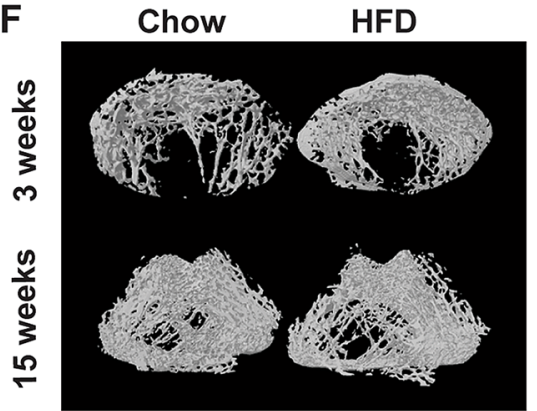

I

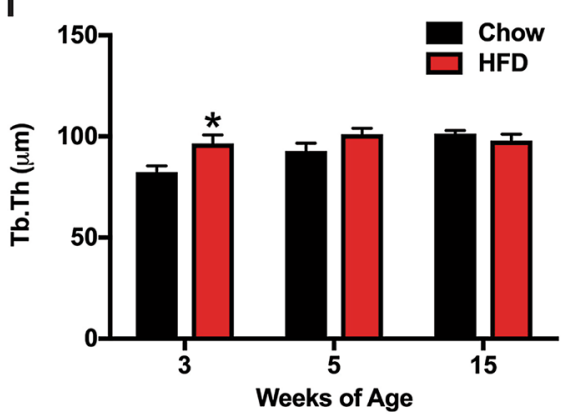

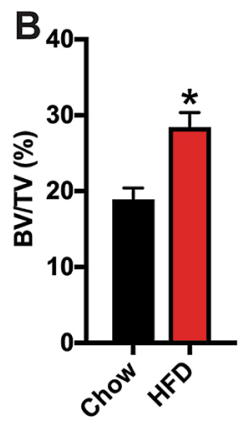
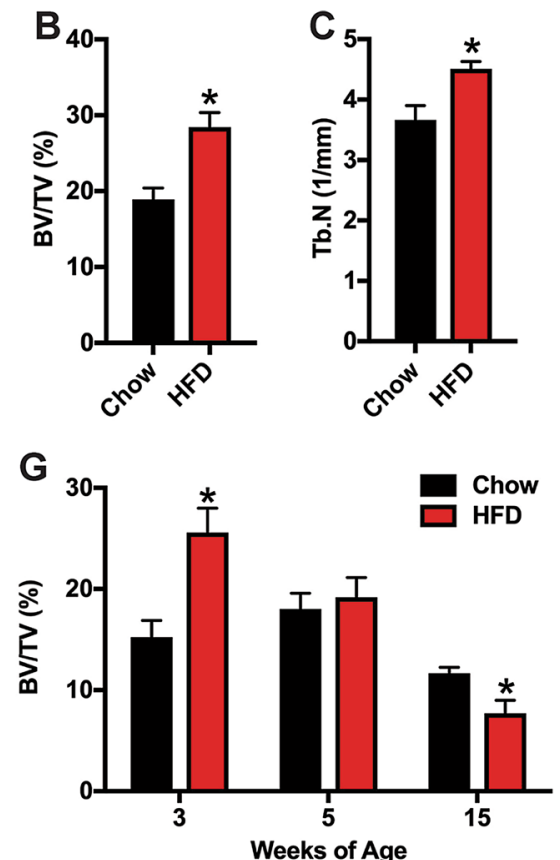

J
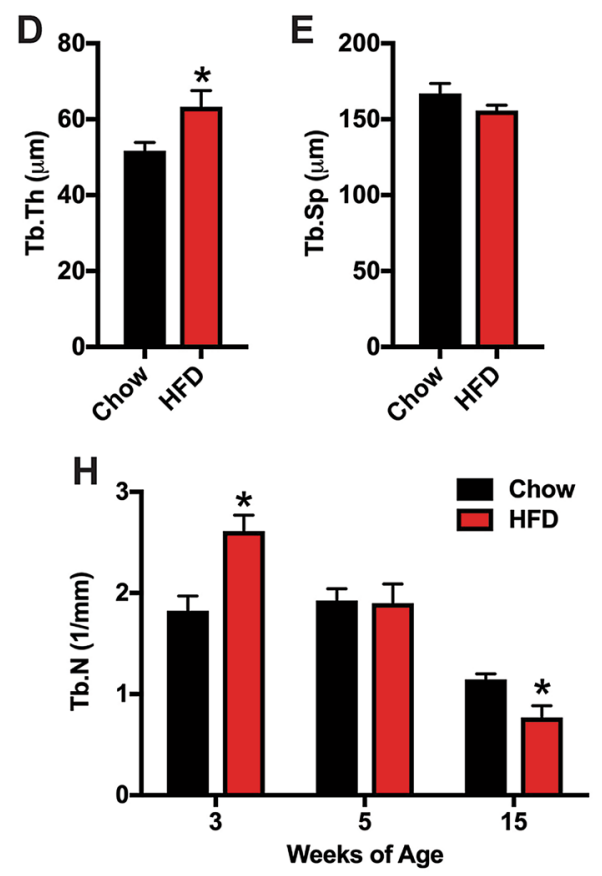

Fig. 3 MicroCT analysis of trabecular bone in male offspring. a Representative microCT images of the distal femur at postnatal week 0 (P1) of the male offspring of dams fed a Chow or HFD. b-e Quantification of trabecular bone volume/tissue volume (b, BV/TV), trabecular number (c, Tb.N), trabecular thickness (d, Tb.Th) and trabecular separation (e, Tb.Sp) in the distal femur ( $n=8-15$ rats/group) at postnatal week 0 . $\mathbf{f}$ Representative microCT images of the distal femur at postnatal week 3 and 15 of the male offspring of dams fed a Chow or HFD. g-j Quantification of trabecular bone volume/tissue volume (g, BV/TV), trabecular number (h, Tb.N), trabecular thickness (i, Tb.Th) and trabecular separation (j, Tb.Sp) in the distal femur ( $n=8-15$ rats/ group) at postnatal weeks 3,5 and 15 . All results are expressed as mean \pm SEM, $* p<0.05$ and HFD dams exhibited similar capacity for mineralized nodule formation (Fig. 5a) and expression of Runx2, Collal, and Bglap (Fig. 5b) after 21 days of osteogenic differentiation. Interestingly, the expression of Tnfsf 11 , encoding RANKL, was significantly increased in stromal cell cultures from offspring of HFD dams, while the expression of Tnfsf $11 \mathrm{~b}$, encoding the soluble inhibitor Osteoprotegerin, was not affected. Thus, maternal HFD feeding appears to lead to a cell autonomous increase in osteoblastic signaling to stimulate osteoclastic bone resorption.

Marked differences in osteoclastic differentiation after M-CSF and RANKL stimulation were evident in cultures of osteoclast precursors isolated from the offspring of Chow and HFD dams. TRAP staining on day 5 of in vitro differentiation revealed that cells isolated from offspring of HFD dams formed larger osteoclasts with more numerous nuclei than those isolated from the offspring of Chow dams (Fig. 5c, d). Consistent with this finding, the expression of Tnfrsl la, encoding RANK, and Dcstamp, a mediator of osteoclastic cell fusion, were significantly increased in cells cultures isolated from the offspring of HFD dams when compared to those from the offspring of Chow dams (Fig. 5e). The increase in RANK expression led us to speculate that maternal HFD feeding led to an increase in the sensitivity of osteoclastic precursors to RANKL. To test this, we performed a dose-response study wherein osteoclast precursors from the adult male offspring of Chow or 

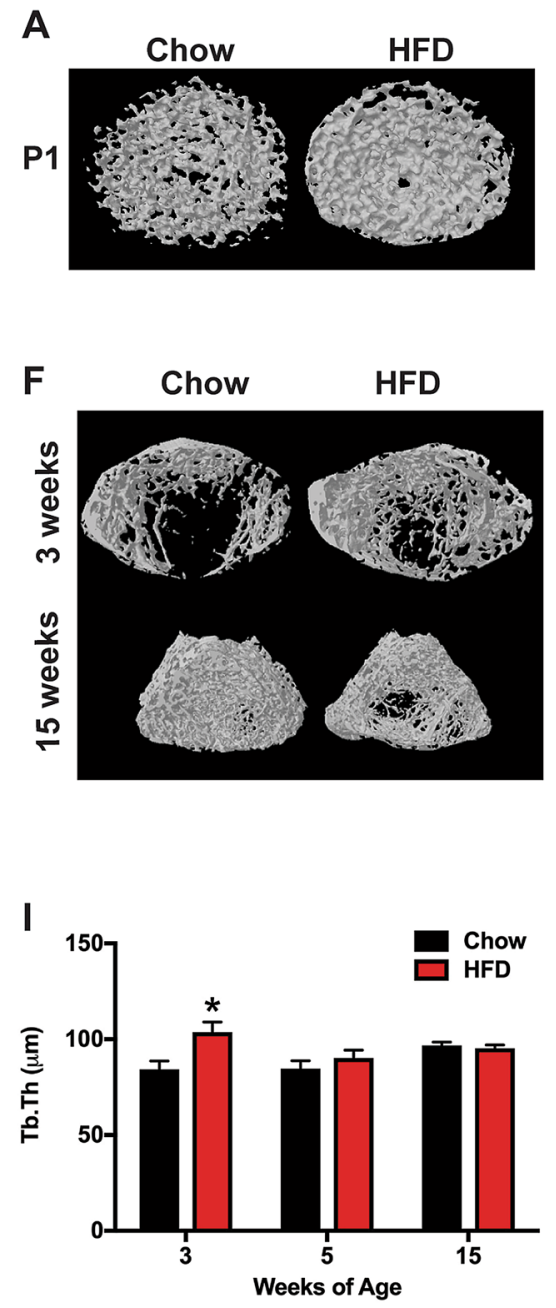
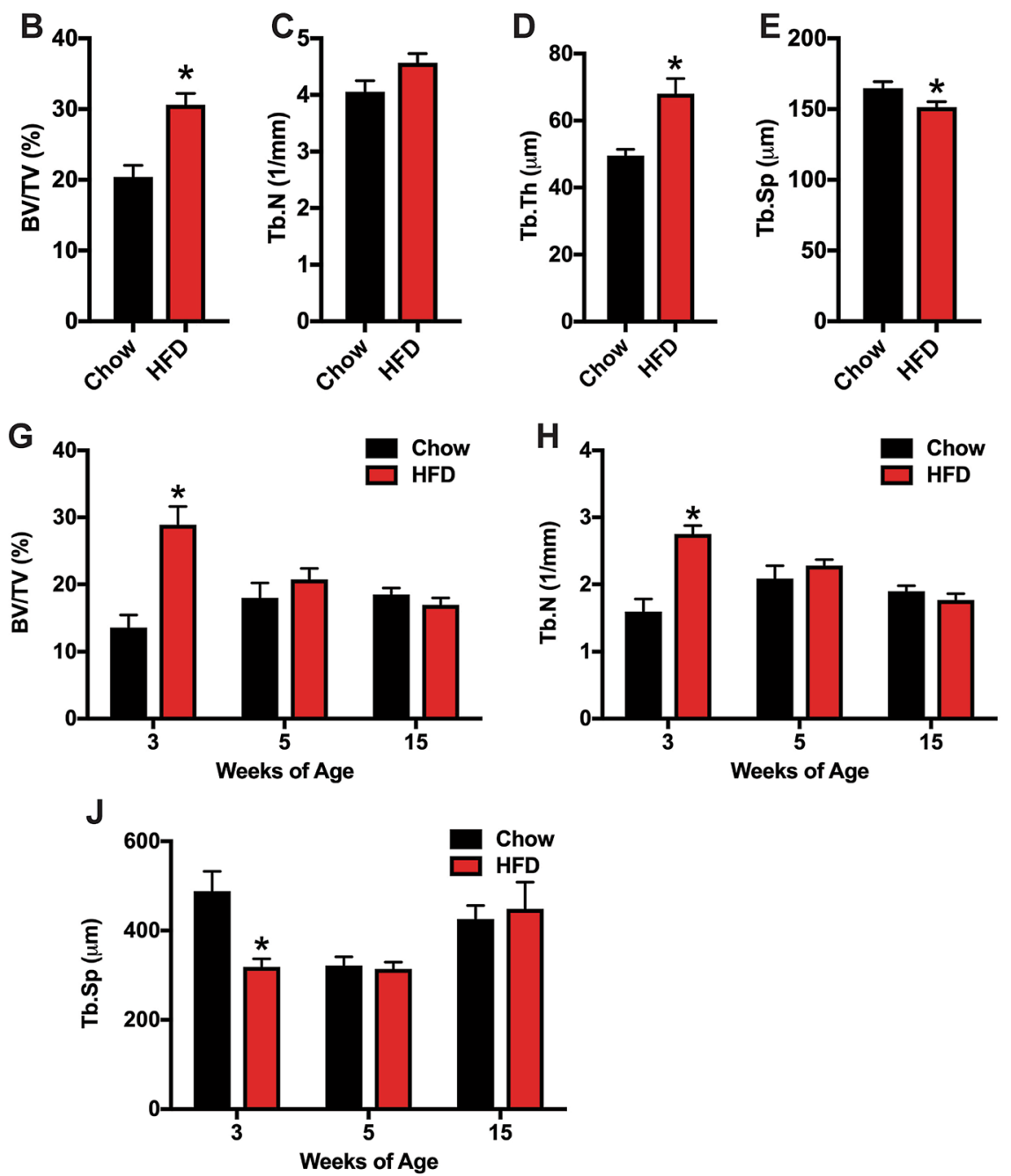

Fig. 4 MicroCT analysis of trabecular bone in female offspring. a Representative microCT images of the distal femur at postnatal week 0 (P1) of the female offspring of dams fed a Chow or HFD. b-e Quantification of trabecular bone volume/tissue volume (b, BV/TV), trabecular number (c, Tb.N), trabecular thickness (d, Tb.Th) and trabecular separation (e, Tb.Sp) in the distal femur ( $n=8-15$ rats/group) at postnatal week 0 . f Representative microCT images of the distal femur at postnatal week 3 and 15 of the female offspring of dams fed a Chow or HFD. $\mathbf{g}-\mathbf{j}$ Quantification of trabecular bone volume/tissue volume (g, BV/TV), trabecular number (h, Tb.N), trabecular thickness (i, Tb.Th) and trabecular separation (j, Tb.Sp) in the distal femur ( $n=8-14$ rats/group) at postnatal weeks 3, 5 and 15. All results are expressed as mean \pm SEM, ${ }^{*} p<0.05$
HFD dams were treated with M-CSF (50 ng/ml) and 0, 0.5, 5 , or $50 \mathrm{ng} / \mathrm{ml}$ RANKL for 5 days. No osteoclasts were evident in the 0 or $0.5 \mathrm{ng} / \mathrm{ml}$ RANKL treatment groups (Fig. 5f). At $5 \mathrm{ng} / \mathrm{ml}$ RANKL, robust differentiation of TRAP + osteoclasts was evident in precursors isolated from the offspring of HFD dams while few multi-nucleated TRAP + cells were evident in cultures from offspring of Chow dams (Fig. 5f, g). Altogether, these data suggest that maternal HFD leads to a sensitization of osteoclast precursors to RANKL as well as an increase in osteoblast RANKL production that in turn leads to a state of accelerated age-related bone loss in male offspring.

\section{Discussion}

It has become clear that adverse maternal exposures during prenatal and early postnatal life can impair offspring health across the lifespan and across physiological systems. With the dramatic rise in worldwide obesity rates and globalization of the high-fat Western-style diet model, recent attention has turned to the consequences of maternal exposure to HFD and overnutrition on offspring [4]. Maternal overnutrition has been associated with increased risk for a range of adverse health effects in offspring [22], and emerging clinical and preclinical evidence suggests it 
Table 3 Static and dynamic histomorphometry

\begin{tabular}{|c|c|c|}
\hline \multirow[t]{2}{*}{ Bone parameter } & \multicolumn{2}{|l|}{ Dam diet } \\
\hline & Chow $(n=7)$ & High fat $(n=6)$ \\
\hline \multicolumn{3}{|l|}{ Bone formation } \\
\hline Osteoid volume/bone volume (OV/BV; \%) & $0.22 \pm 0.04$ & $0.41 \pm 0.09$ \\
\hline Osteoid surface/bone surface (OS/BS; \%) & $2.88 \pm 0.45$ & $5.25 \pm 1.37$ \\
\hline Osteoid thickness (O.Th; $\mu \mathrm{m})$ & $2.06 \pm 0.30$ & $2.19 \pm 0.24$ \\
\hline Osteoblast surface/bone surface (Ob.S/BS; \%) & $2.27 \pm 0.46$ & $3.87 \pm 0.76$ \\
\hline Osteoblast number/bone perimeter (NOb/BPm; no./100 mm) & $170.13 \pm 37.70$ & $285.91 \pm 58.62$ \\
\hline \multicolumn{3}{|l|}{ Bone erosion } \\
\hline Erosion surface/bone surface (ES/BS; \%) & $1.51 \pm 0.34$ & $3.65 \pm 0.77 *$ \\
\hline Osteoclast surface (Oc.S/BS; \%) & $1.40 \pm 0.75$ & $3.54 \pm 0.89 *$ \\
\hline Osteoclast number/bone perimeter (NOc/BPm; no./100 mm) & $36.13 \pm 9.03$ & $101.59 \pm 27.61 *$ \\
\hline \multicolumn{3}{|l|}{ Bone dynamics } \\
\hline Mineral apposition rate (MAR; $\mu \mathrm{m} /$ day) & $1.29 \pm 0.11$ & $1.49 \pm 0.13$ \\
\hline Mineralizing surface/bone surface (MS/BS; \%) & $16.54 \pm 1.43$ & $21.07 \pm 2.59$ \\
\hline Bone formation rate/bone surface (BFR/BS; $\mathrm{mm}^{3} / \mathrm{cm}^{2} /$ year) & $7.98 \pm 1.32$ & $12.02 \pm 2.32$ \\
\hline Mineralization lag time (Mlt; day) & $0.32 \pm 0.06$ & $0.41 \pm 0.13$ \\
\hline Osteoid maturation time (Omt; day) & $1.61 \pm 0.20$ & $1.56 \pm 0.23$ \\
\hline
\end{tabular}

may also exert long term consequences on skeletal health $[6,8,9,11,12]$. In this study, we used an established model of maternal HFD feeding [13, 14, 23] to examine both short and long term effects on the skeletal health of offspring. Using the same paradigm [17-19], we previously reported that both male and female HFD offspring are similar in body weight at birth, but have increased body weight by P7 and have greater adiposity by P10. Glucose tolerance is impaired in HFD offspring by 3 weeks of age, the earliest age tested, and HFD offspring are hyperleptinemic throughout the early postnatal period, but are leptin resistant and have deficits in hypothalamic leptin signaling when tested at P10 and 3 weeks of age [17-19].

In young offspring (through 3 weeks of age), maternal HFD feeding resulted in a marked increase in trabecular bone volume. Though not directly examined, this phenotype is almost assuredly due to an increase in osteoblast activity, since bone modeling is most active during this period of rapid growth [24-26]. We speculate that this early effect is due to nutritional effects as our previous studies indicated that dams fed a HFD spent more time nursing that those fed a chow diet and the offspring of HFD dams consume more milk than those of chow dams [23]. The milk of HFD fed dams also tended to have a higher concentration of fat and protein than that of chow fed dams resulting in greater energy content [23]. Although maternal bone remodeling is normally greater during pregnancy and lactation due to increased calcium requirements by the developing fetus and neonate, maternal bone loss appears to have been exacerbated in HFD dams and this could be a consequence of greater demand for milk by HFD offspring.

After 3 weeks of age, the male and to a lesser extent female offspring of HFD fed dams exhibited greater bone loss than the offspring of chow fed dams. The cellular basis for this phenotype in our study differs from previous studies that used both mice and rats and demonstrated maternal HFD feeding negatively impacted osteoblast performance in offspring [11, 12, 27]. As an example, data from the Shankar laboratory identified an increase in the senescence associated secretory phenotype in fetal calvarial osteoblasts isolated from the progeny of HFD fed rat dams [12]. In our study, we only observed a change in the abundance of osteoclasts in vivo and enhanced osteoclast differentiation in vitro. In all likelihood, the trends towards increases in osteoid parameters and the mineralizing surface are due to the coupling of osteoblast activity to heightened osteoclastic activity. The main difference between our study and the others is the length of maternal challenge with a HFD. In previous studies $[11,12,27]$, dams were fed a HFD for 4 to 12 weeks prior to breeding and then through the weaning of pups. Here, dams were only challenged with HFD from gestational day 2 through weaning. These differences suggest the length of exposure to nutritional stressors dictates which cell populations involved in bone remodeling are affected.

The length of maternal exposure to HFD is likely to result in differences in the development of metabolic disturbances in dams that may then affect offspring. Our previous studies showed that HFD exposure from gestational day 2 to 

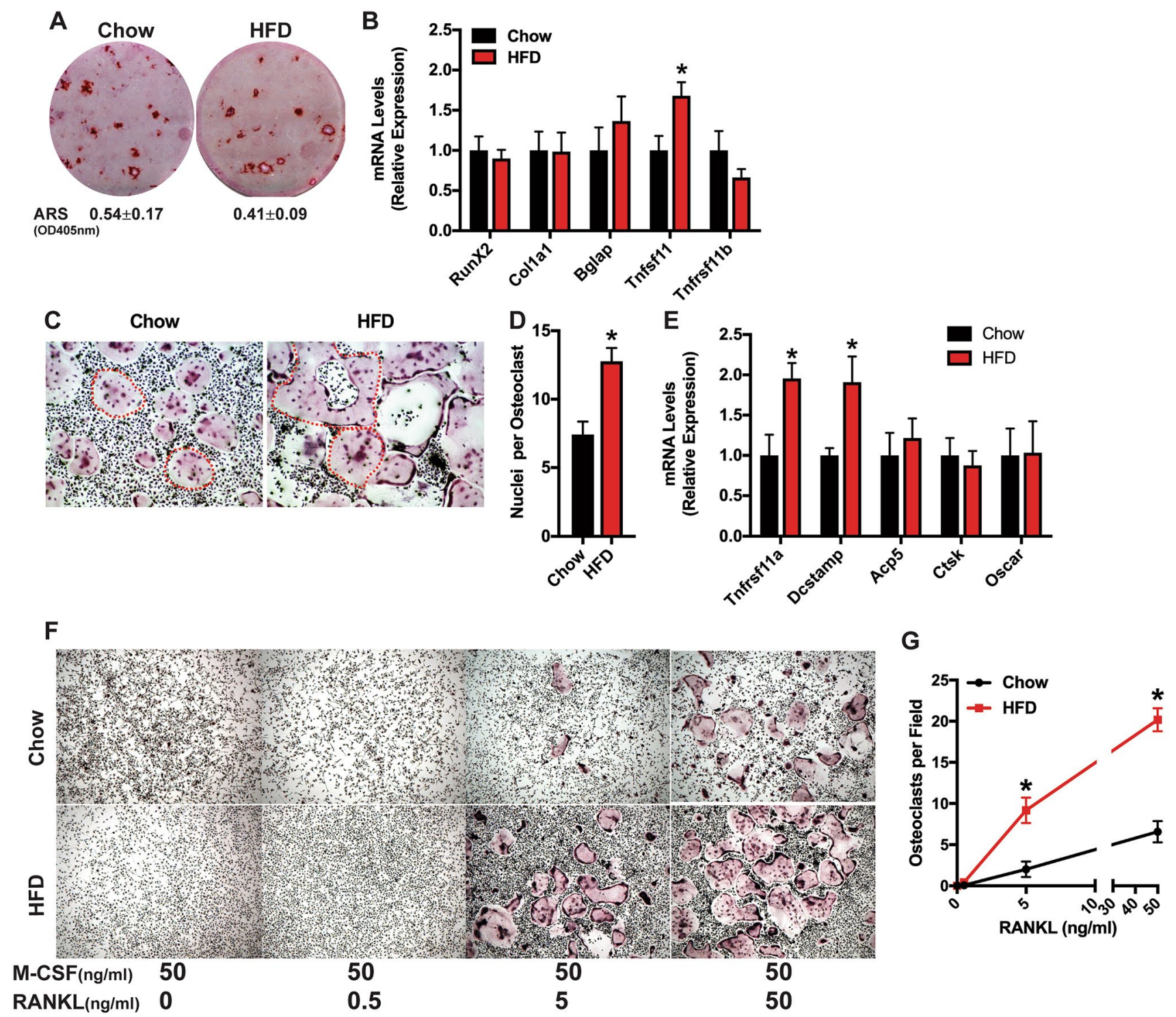

lined in red $(\mathbf{c}, \times 6.4$ original magnification $)$, assessment of nuclei per

Fig. 5 Maternal high fat diet enhances in vitro osteoclastogenesis in offspring. a, b Bone marrow stromal cells isolated from the offspring of dams fed a Chow or HFD were differentiated under osteogenic conditions for 21 days. Alizarin red staining (a) and qPCR (b) were used to assess differentiation. Quantification of Alizarin red staining after extraction is shown under the images. c-e Osteoclast precursors isolated from the offspring of dams fed a Chow or HFD were differentiated in the presence of M-CSF (50 ng/ml) and RANKL (50 ng/ $\mathrm{ml}$ ) for 5 days. TRAP staining with two osteoclasts per imaged out-

weaning results in changes to several metabolic indices in dams. Increases in body weight relative to Chow-fed dams is not consistently observed in HFD fed dams, but adipose tissue mass is increased by birth and coupled with the development of hyperleptinemia by G10 when compared to Chowfed dams [14, 18, 19]. In addition, HFD fed dams exhibit impaired glucose tolerance and decreased plasma adiponectin as early as G14 [14]. We did not make direct measurements of dam metabolism during gestation or lactation here osteoclast (d) and qPCR (e) were used to assess differentiation. f, $\mathbf{g}$ Osteoclast precursors isolated from the offspring of dams fed a Chow or HFD were differentiated in the presence of M-CSF $(50 \mathrm{ng} / \mathrm{ml})$ and the indicated concentration of RANKL for 5 days. TRAP staining $(\mathbf{f}, \times 4$ original magnification) and quantification of osteoclasts per field were used to assess differentiation. All results are expressed as mean \pm SEM, $* p<0.05$. In vitro studies were replicated in cell cultures isolated from 4 to 7 rats

as blood collection or glucose tolerance testing would introduce additional stressors as confounding variables, but HFD dams had higher plasma leptin levels at weaning suggesting that they had greater adiposity. Longer periods of maternal HFD exposure than employed here are likely to result in more severe metabolic changes. For example, beginning maternal HFD access several weeks prior to conception, as conducted in the studies described above, results in maternal obesity and metabolic derangement [28, 29]. In addition, 
preconception obesity may also alter oocyte metabolism, morphology and maturation [30,31]. Such differences in model design - preconception obesity versus perinatal HFD exposure-could thus alter the maternal metabolic milieu and subsequent severity and trajectory of the developmental environment experienced by the offspring. Nevertheless, the consistent theme regardless of the model used is that maternal diet has a significant and persistent consequence on offspring's bone biology and health, even if the offspring themselves did not consume the HFD. In fact, weaning on to a HFD or provision of HFD to adult offspring would likely exacerbate bone remodeling and accelerate disease, as has been reported for other phenotypes affected by maternal HFD [17, 32, 33].

It is not surprising that the ultimate offspring phenotype is dependent on the timing of maternal dietary manipulation, whether the dam's exposure occurs throughout life prior to pregnancy or only during the immediate perinatal period after fertilization has occurred. In each case the "insult" occurs at different developmentally sensitive time points for the offspring whether it is as a germ cell, early embryo, fetus, or neonate. Such outcomes from different models provide clues that may be leveraged to determine how maternal diet or metabolic state affects different stages of bone development (i.e. differentiation of precursors, longitudinal growth, coupling of formation to resorption, etc.) depending on the specific exposure windows.

Determining the mechanism by which maternal HFD feeding results in increased osteoclastogenesis will require additional study. This effect could be secondary to alterations in metabolic hormones like leptin and insulin that influence bone remodeling, and/or changes in the activity of osteocytes, which transgenic mouse models indicate are the dominant source of RANKL production in vivo [34, 35] and trigger targeted resorption in response to apoptotic signals [36]. Consistent with this later possibility, our in vitro data suggests increases in bone resorption are at least partially due to cell autonomous effects on osteoblasts and osteoclasts. Osteoblasts differentiated from bone marrow stromal cells isolated from the offspring of HFD fed dams exhibited an increase in RANKL expression even though they did not exhibit an impairment in markers of differentiation potential. These data suggest osteoblastic signaling to osteoclasts is increased. On the other hand, osteoclasts exhibited an increase in RANK expression and precursors exhibited an increase in sensitivity to RANKL. This effect could be mediated by epigenetic changes that influence the machinery that confers sensitivity to differentiation inducing signals. There is a growing appreciation of epigenetic control both in normal bone biology and in the onset and progression of musculoskeletal disease [37-39]. Epigenetic profiles that ultimately influence gene, cell, tissue and organ function are established during the perinatal period. Recent findings reveal the role of DNA methylation in control of Wnt, RANK/RANKL, and other key signaling pathways, as well as epigenetic regulation of osteoblast and osteoclast differentiation [39]. Thus, maternal diet could alter epigenetic histone modifications, DNA methylation, and non-coding RNAs in offspring to confer susceptibility to pathologic bone remodeling that persists throughout life.

In summary, our study indicates that a maternal dietary insult is sufficient to produce long term defects in skeletal remodeling that may ultimately lead to a predisposition for the development of osteopenia/osteoporosis. Future work will be directed toward understanding the cell autonomous mechanism by which maternal HFD instructs an increase in osteoclast development as well as the exploration of therapeutic interventions that offset the detrimental effects of maternal stressors.

Acknowledgements The authors are grateful to Marie-Claude Faugere for assistance with histomorphometric analyses. The studied in this manuscript were supported by NIH HD093338 (S. G. K.), NIH DK099134 (R. C. R), VA BX003742 (R.C.R), and NIH MH108944 (K. L. K. T.)

Funding The funding was supported by National Institutes of Health (Grant Nos. HD093338 and MH108944), National Institute of Diabetes and Digestive and Kidney Diseases (Grant No. DK099134) and U.S. Department of Veterans Affairs (Grant No. BX003742).

\section{Compliance with Ethical Standards}

Conflict of Interest Priyanka Kushwaha, Seva G. Khambadkone, Mengni Li, Ethan J. Goodman, Nandini Aravindan declare that they have no conflicts of interest, financial or otherwise, are declared by the authors.

Human and Animal Rights and Informed Consent All animal procedures were approved by the Animal Care and Use Committee of the Johns Hopkins University School of Medicine. No human subjects or human tissue/cells were used in the manuscript, so there is no informed consent statement.

Open Access This article is licensed under a Creative Commons Attribution 4.0 International License, which permits use, sharing, adaptation, distribution and reproduction in any medium or format, as long as you give appropriate credit to the original author(s) and the source, provide a link to the Creative Commons licence, and indicate if changes were made. The images or other third party material in this article are included in the article's Creative Commons licence, unless indicated otherwise in a credit line to the material. If material is not included in the article's Creative Commons licence and your intended use is not permitted by statutory regulation or exceeds the permitted use, you will need to obtain permission directly from the copyright holder. To view a copy of this licence, visit http://creativecommons.org/licenses/by/4.0/. 


\section{References}

1. Siddiqui JA, Partridge NC (2016) Physiological bone remodeling: systemic regulation and growth factor involvement. Physiology 31(3):233-245. https://doi.org/10.1152/physiol.00061.2014

2. Richards JB, Rivadeneira F, Inouye M, Pastinen TM, Soranzo N, Wilson SG, Andrew T, Falchi M, Gwilliam R, Ahmadi KR, Valdes AM, Arp P, Whittaker P, Verlaan DJ, Jhamai M, Kumanduri V, Moorhouse M, van Meurs JB, Hofman A, Pols HA, Hart D, Zhai G, Kato BS, Mullin BH, Zhang F, Deloukas P, Uitterlinden AG, Spector TD (2008) Bone mineral density, osteoporosis, and osteoporotic fractures: a genome-wide association study. Lancet 371(9623):1505-1512. https://doi.org/10.1016/S0140 $-6736(08) 60599-1$

3. Styrkarsdottir U, Halldorsson BV, Gretarsdottir S, Gudbjartsson DF, Walters GB, Ingvarsson T, Jonsdottir T, Saemundsdottir J, Center JR, Nguyen TV, Bagger Y, Gulcher JR, Eisman JA, Christiansen C, Sigurdsson G, Kong A, Thorsteinsdottir U, Stefansson K (2008) Multiple genetic loci for bone mineral density and fractures. N Engl J Med 358(22):2355-2365. https://doi.org/10.1056/ NEJMoa0801197

4. Khambadkone SG, Cordner ZA, Tamashiro KLK (2020) Maternal stressors and the developmental origins of neuropsychiatric risk. Front Neuroendocrinol 57:100834. https://doi.org/10.1016/j.yfrne .2020 .100834

5. Boersma GJ, Bale TL, Casanello P, Lara HE, Lucion AB, Suchecki D, Tamashiro KL (2014) Long-term impact of early life events on physiology and behaviour. J Neuroendocrinol 26(9):587-602. https://doi.org/10.1111/jne.12153

6. Petersen SB, Rasmussen MA, Olsen SF, Vestergaard P, Molgaard C, Halldorsson TI, Strom M (2015) Maternal dietary patterns during pregnancy in relation to offspring forearm fractures: prospective study from the Danish National Birth Cohort. Nutrients 7(4):2382-2400. https://doi.org/10.3390/nu7042382

7. Cole ZA, Gale CR, Javaid MK, Robinson SM, Law C, Boucher BJ, Crozier SR, Godfrey KM, Dennison EM, Cooper C (2009) Maternal dietary patterns during pregnancy and childhood bone mass: a longitudinal study. J Bone Miner Res 24(4):663-668. https ://doi.org/10.1359/jbmr.081212

8. Liang C, Oest ME, Jones JC, Prater MR (2009) Gestational high saturated fat diet alters C57BL/6 mouse perinatal skeletal formation. Birth Defects Res B 86(5):362-369. https://doi.org/10.1002/ bdrb.20204

9. Liang C, Oest ME, Prater MR (2009) Intrauterine exposure to high saturated fat diet elevates risk of adult-onset chronic diseases in C57BL/6 mice. Birth Defects Res B 86(5):377-384. https://doi. org/10.1002/bdrb.20206

10. Knight BS, Pennell CE, Adamson SL, Lye SJ (2007) The impact of murine strain and sex on postnatal development after maternal dietary restriction during pregnancy. J Physiol 581(Pt 2):873-881. https://doi.org/10.1113/jphysiol.2006.126573

11. Chen JR, Zhang J, Lazarenko OP, Kang P, Blackburn ML, Ronis MJ, Badger TM, Shankar K (2012) Inhibition of fetal bone development through epigenetic down-regulation of HoxA10 in obese rats fed high-fat diet. FASEB J 26(3):1131-1141. https://doi. org/10.1096/fj.11-197822

12. Chen JR, Lazarenko OP, Blackburn ML, Rose S, Frye RE, Badger TM, Andres A, Shankar K (2016) Maternal obesity programs senescence signaling and glucose metabolism in osteo-progenitors from rat and human. Endocrinology 157(11):4172-4183. https:// doi.org/10.1210/en.2016-1408

13. Cordner ZA, Khambadkone SG, Boersma GJ, Song L, Summers TN, Moran TH, Tamashiro KLK (2019) Maternal high-fat diet results in cognitive impairment and hippocampal gene expression changes in rat offspring. Exp Neurol 318:92-100. https://doi. org/10.1016/j.expneurol.2019.04.018

14. Song L, Sun B, Boersma GJ, Cordner ZA, Yan J, Moran TH, Tamashiro KLK (2017) Prenatal high-fat diet alters placental morphology, nutrient transporter expression, and mtorc1 signaling in rat. Obesity 25(5):909-919. https://doi.org/10.1002/oby.21821

15. Bouxsein ML, Boyd SK, Christiansen BA, Guldberg RE, Jepsen KJ, Muller R (2010) Guidelines for assessment of bone microstructure in rodents using micro-computed tomography. J Bone Miner Res 25(7):1468-1486. https://doi.org/10.1002/jbmr.141

16. Dempster DW, Compston JE, Drezner MK, Glorieux FH, Kanis JA, Malluche H, Meunier PJ, Ott SM, Recker RR, Parfitt AM (2013) Standardized nomenclature, symbols, and units for bone histomorphometry: a 2012 update of the report of the ASBMR histomorphometry nomenclature committee. J Bone Miner Res 28(1):2-17. https://doi.org/10.1002/jbmr.1805

17. Tamashiro KL, Terrillion CE, Hyun J, Koenig JI, Moran TH (2009) Prenatal stress or high-fat diet increases susceptibility to diet-induced obesity in rat offspring. Diabetes 58(5):1116-1125. https://doi.org/10.2337/db08-1129

18. Sun B, Song L, Tamashiro KL, Moran TH, Yan J (2014) Large litter rearing improves leptin sensitivity and hypothalamic appetite markers in offspring of rat dams fed high-fat diet during pregnancy and lactation. Endocrinology 155(9):3421-3433. https:// doi.org/10.1210/en.2014-1051

19. Sun B, Purcell RH, Terrillion CE, Yan J, Moran TH, Tamashiro KL (2012) Maternal high-fat diet during gestation or suckling differentially affects offspring leptin sensitivity and obesity. Diabetes 61(11):2833-2841. https://doi.org/10.2337/db11-0957

20. Hunziker EB, Schenk RK (1989) Physiological mechanisms adopted by chondrocytes in regulating longitudinal bone growth in rats. J Physiol 414:55-71. https://doi.org/10.1113/jphysiol.1989. sp017676

21. Roach HI, Mehta G, Oreffo RO, Clarke NM, Cooper C (2003) Temporal analysis of rat growth plates: cessation of growth with age despite presence of a physis. J Histochem Cytochem 51(3):373-383. https://doi.org/10.1177/002215540305100312

22. Godfrey KM, Reynolds RM, Prescott SL, Nyirenda M, Jaddoe VW, Eriksson JG, Broekman BF (2017) Influence of maternal obesity on the long-term health of offspring. Lancet Diabetes Endocrinol 5(1):53-64. https://doi.org/10.1016/S2213 -8587(16)30107-3

23. Purcell RH, Sun B, Pass LL, Power ML, Moran TH, Tamashiro KL (2011) Maternal stress and high-fat diet effect on maternal behavior, milk composition, and pup ingestive behavior. Physiol Behav 104(3):474-479. https://doi.org/10.1016/j.physb eh.2011.05.012

24. Kimmel DB, Jee WS (1980) Bone cell kinetics during longitudinal bone growth in the rat. Calcif Tissue Int 32(2):123-133. https:// doi.org/10.1007/bf02408531

25. Kimmel DB, Jee WS (1980) A quantitative histologic analysis of the growing long bone metaphysis. Calcif Tissue Int 32(2):113122. https://doi.org/10.1007/bf02408530

26. Frost HM, Jee WS (1992) On the rat model of human osteopenias and osteoporoses. Bone Miner 18(3):227-236. https://doi. org/10.1016/0169-6009(92)90809-r

27. Shi Y, Saben JL, He G, Moley KH, Long F (2020) Diet-induced metabolic dysregulation in female mice causes osteopenia in adult offspring. J Endocr Soc. https://doi.org/10.1210/jendso/bvaa028

28. Taylor PD, Matthews PA, Khan IY, Rees D, Itani N, Poston L (2018) Generation of maternal obesity models in studies of developmental programming in rodents. Methods Mol Biol 1735:167199. https://doi.org/10.1007/978-1-4939-7614-0_9

29. Khambadkone SG, Cordner ZA, Tamashiro KLK (2020) Maternal stressors and the developmental origins of neuropsychiatric 
risk. Front Neuroendocrinol. https://doi.org/10.1016/j.yfrne .2020 .100834

30. Reynolds KA, Boudoures AL, Chi MM, Wang Q, Moley KH (2015) Adverse effects of obesity and/or high-fat diet on oocyte quality and metabolism are not reversible with resumption of regular diet in mice. Reprod Fertil Dev 27(4):716-724. https:// doi.org/10.1071/RD14251

31. Luzzo KM, Wang Q, Purcell SH, Chi M, Jimenez PT, Grindler N, Schedl T, Moley KH (2012) High fat diet induced developmental defects in the mouse: oocyte meiotic aneuploidy and fetal growth retardation/brain defects. PLoS ONE 7(11):e49217. https://doi. org/10.1371/journal.pone.0049217

32. Simar D, Chen H, Lambert K, Mercier J, Morris MJ (2012) Interaction between maternal obesity and post-natal over-nutrition on skeletal muscle metabolism. Nutr Metab Cardiovasc Dis 22(3):269-276. https://doi.org/10.1016/j.numecd.2010.11.007

33. Rajia S, Chen H, Morris MJ (2010) Maternal overnutrition impacts offspring adiposity and brain appetite markers-modulation by postweaning diet. J Neuroendocrinol 22(8):905-914. https ://doi.org/10.1111/j.1365-2826.2010.02005.x

34. Xiong J, Onal M, Jilka RL, Weinstein RS, Manolagas SC, O’Brien CA (2011) Matrix-embedded cells control osteoclast formation. Nat Med 17(10):1235-1241. https://doi.org/10.1038/nm.2448

35. Nakashima T, Hayashi M, Fukunaga T, Kurata K, Oh-Hora M, Feng JQ, Bonewald LF, Kodama T, Wutz A, Wagner EF,
Penninger JM, Takayanagi H (2011) Evidence for osteocyte regulation of bone homeostasis through RANKL expression. Nat Med 17(10):1231-1234. https://doi.org/10.1038/nm.2452

36. Plotkin LI (2014) Apoptotic osteocytes and the control of targeted bone resorption. Curr Osteoporos Rep 12(1):121-126. https://doi. org/10.1007/s11914-014-0194-3

37. Yi SJ, Lee H, Lee J, Lee K, Kim J, Kim Y, Park JI, Kim K (2019) Bone remodeling: histone modifications as fate determinants of bone cell differentiation. Int J Mol Sci. https://doi.org/10.3390/ ijms 20133147

38. Bellavia D, De Luca A, Carina V, Costa V, Raimondi L, Salamanna F, Alessandro R, Fini M, Giavaresi G (2019) Deregulated miRNAs in bone health: epigenetic roles in osteoporosis. Bone 122:52-75. https://doi.org/10.1016/j.bone.2019.02.013

39. Husain A, Jeffries MA (2017) Epigenetics and bone remodeling. Curr Osteoporos Rep 15(5):450-458. https://doi.org/10.1007/ s11914-017-0391-y

Publisher's Note Springer Nature remains neutral with regard to jurisdictional claims in published maps and institutional affiliations. 